\title{
Traffic Conflict Techniques in the Czech Republic
}

\author{
Josef Kocourek* \\ Czech Technical University in Prague Faculty of Transportation Science, Czech Republic. \\ *Corresponding author: kocoujos@fd.cvut.cz
}

DOI: $10.2478 / \mathrm{V} 10158-010-0017-\mathrm{X}$

\begin{abstract}
This article deals mainly with defining a way of monitoring traffic conflicts. This issue is only one part of a greater safety analysis, or risk determination process for a particular location. Based on the described procedures it is possible to apply the methodology also to more complicated nodes, such as level crossings, sections of towns, residential areas, or rural areas. After the survey and the collection of a necessary amount of transport and engineering data, the determination of a relative number for the "almostaccident rate" (number of calculated vehicles for a selected time period and the number of traffic conflicts - so-called almost-accidents) may be calculated.
\end{abstract}

KEY WORDS: Traffic conflict techniques, monitoring traffic conflicts, safety analysis, accident

\section{INTRODUCTION}

In most road safety studies, analysts use the information contained in accident reports to identify and understand the failures of the road system and then propose appropriate corrective action. While these analyses are essential, it is well recognized that accident data suffer from a number of shortcomings and need to be complemented by field observations in order to improve the accuracy of safety diagnoses. The traffic conflict technique (TCT) was originally developed by researchers from General Motors Laboratories who wanted to investigate whether GM cars were driven differently than others (Perkins and Harris, 1968). Since then, several variants of the original TCT have been proposed. Almost all traffic conflict techniques take into consideration the need, for at least one road user, to take evasive action in order to avoid a collision (braking, swerving, accelerating, or a combination of these manoeuvres). A well-accepted definition of traffic conflict is:

"an observable situation in which two or more road users approach each other in space and time to such an extent that there is a risk of collision if their movements remain unchanged".

\section{METHODS}

My research is focused on traffic conflicts as an alternative to accident data. Conflicts occur far more frequently in traffic and can include the whole range of incidences where the actual accident is just at one end of the scale. Techniques range from subjective to the more objective where conflicts are rated by measurements such as time to collision or post encroachment time. Two accident locations are analyzed in my dissertation work. This research advances the research of Dr. Folprecht (Ostrava).

I applied the traffic conflict technique to estimate the traffic safety at unsignalized intersections (roundabouts) and an index measure was developed to summarize the conflict risk at intersections. This index provides an indication regarding the relative risk of being 
involved in a conflict at an intersection. These research efforts further enhance the usefulness of the traffic conflict technique as a tool to evaluate the safety of intersections. Safety and risks are not only described in mathematical terms, when studying traffic safety, it is also important to obtain knowledge concerning human behavior. Conflict studies are often combined with other types of behavioral studies. In order to estimate the number of accidents from conflict registration, ratios between the number of conflicts and accidents are used, which means that conflicts can also be regarded as a measurement of exposure, as well as an indirect estimate of the number of accidents. At intersections, observation or video-recording methods are used to estimate an exposure database for the number of vehicles or pedestrians using the area. The exposure data can be based on traffic situations, either registered accidents or by using traffic conflict techniques for the area. Combined with traffic counting systems, the accident rate or conflict rate can be expressed as the number of accidents per million vehicles entering the intersection. The traffic conflict technique enables one to study hazards in traffic in an uncomplicated way. It used to be the number of reported accidents at a site that decided whether it should be rebuilt or not. For following traffic conflicts in practice it is necessary to defined four basic questions:

a) Why to analyze traffic conflicts,

b) Conflict severity,

c) Types of traffic conflicts,

d) How to conduct a traffic conflict study.

\section{a) Why to analyze traffic conflicts}

A traffic conflict study can be used:

- to make progress in a safety diagnosis. Traffic conflict studies are particularly useful when accident data suffer from strong limitations (accident reports may be unavailable, the information may be insufficient or unreliable),

- to evaluate the effectiveness of a safety remedy. The main advantage of conflict studies over accident studies is that it is not necessary to wait several years before gathering sufficient data to complete the evaluation. A conflict study can be conducted soon after work has been completed and negated quickly if the anticipated benefits have not been achieved (or if unexpected side effects have been created). In these studies, traffic conflicts need to be observed before and after the implementation of the remedy,

- to compare the safety performance of different road features or traffic rules (e.g., to compare safety at signalized intersections with and without an exclusive left-turn phase).

\section{b) Conflict severity}

Most traffic conflict techniques (TCTs) categorize conflicts based on their severity (e.g., serious or non-serious). Some TCTs use subjective criteria to determine conflict severity; for example, the American manual developed by Parker and Zegeer (1989) identifies serious conflicts based on an array of observations that include vehicle front-end-diving when braking, squealing brakes, etc. Other TCTs determine conflict severity by using more objective criteria (which may, however, be more difficult to measure); for example, the Swedish traffic conflict technique combines the TCT and the vehicle speed to distinguish between serious and non-serious conflicts. 
For the TCTs purposes three levels of conflicts have been defined. For the complex analysis of the studied locality even so-called level 0 and level 4 can be monitored. Thus there are 5 levels altogether (Figure 1). We will now describe the constituent levels of conflict severity.

The first level is level 0 . This level is used for the record of an isolated vehicle merely breaking road traffic rules (in the case of the roundabout this kind of manoeuvre was, for example, not to signalize stream compulsory direction change while leaving the roundabout). The level 1 is assigned to the controlled manoeuvre without any limitation or just with a minor limitation. The example of this level is a conflict between a vehicle which is standing on the pedestrian crossing, for example, because of the traffic jam (this is not yet conflict level 1), and the pedestrian, who would like to use this pedestrian crossing, has to go around the vehicle (see the Figure 1 - level 1).
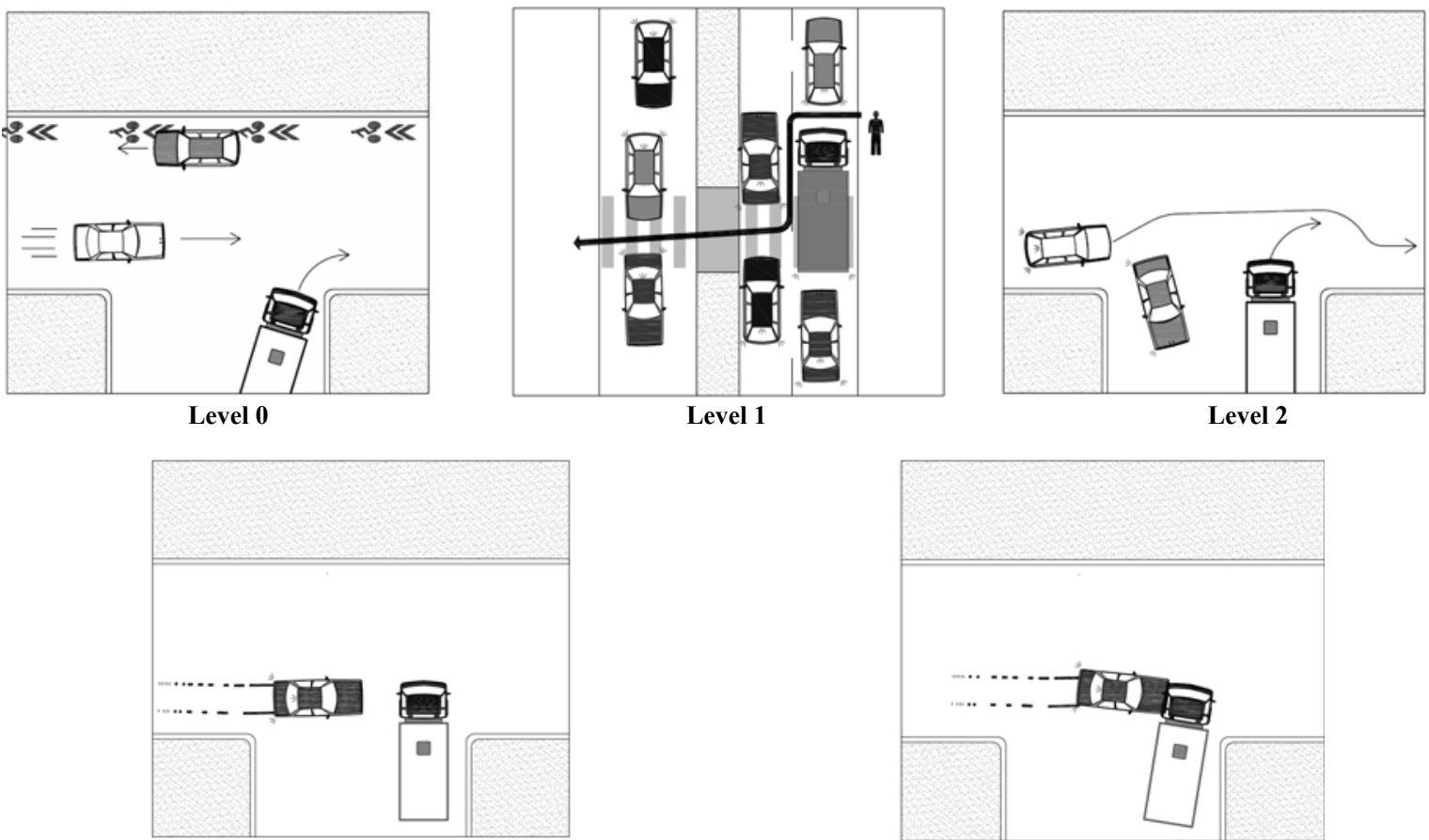

Level 3

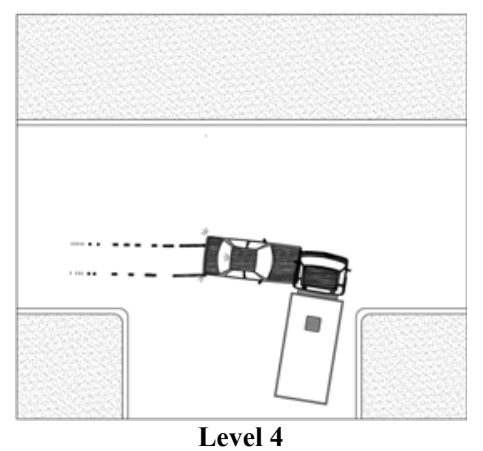

Figure 1: Example of conflict severity.

The difference between level 1 and level 2 is minor. In spite of that, it is necessary to realize, that in some specific situations (the example with pedestrians - see above) it is necessary to divide this kind of conflict into less severe and more severe (level 2). According to the Law n. 361/2000 on traffic on the road the term "not to be allowed to limit" is according to the $\S 2$ article $\mathrm{m}$ ) defined as the duty of the driver not to obstruct another participant of the traffic. Conflict level 3 is assigned to such situations when the road users are threatened and a sharp manoeuvre (loud breaking, supplemented, for example, with sounding the horn) is necessary to avert a traffic accident (according to the Law n. $361 / 2000$ on traffic on the road the term "not to be allowed to endanger"). Level 4 is an accident. An example of a traffic conflict record is below (Figure 2). 


\begin{tabular}{|l|l|l|l|}
\hline \multirow{2}{*}{ Traffic conflict record } & O / B - 1 \\
\cline { 3 - 4 } \multicolumn{2}{|l|}{} & Problem creator / respond - conflict severity \\
\hline \multicolumn{4}{|l|}{ Comment } \\
\hline O & personal vehicle & B & BUS \\
\hline N & small cargo vehicle & T & tramway \\
\hline NT & long vehicle & Ch / C & pedestrian / cyclist \\
\hline
\end{tabular}

Figure 2: Example of traffic conflict record.

\section{c) Types of traffic conflicts}

As in the case of accident analyses, it is quite useful to subdivide traffic conflicts into different categories, based on their type. This allows the preparation of summary tables, graphs and diagrams that facilitate the interpretation of results (comparisons with sites having similar characteristics and the detection of deviant types of traffic conflicts). Our research defined 14 types of conflicts between two vehicles (one of them is on Figure 3 - left) 1 example of secondary conflicts (Figure 3 - right).
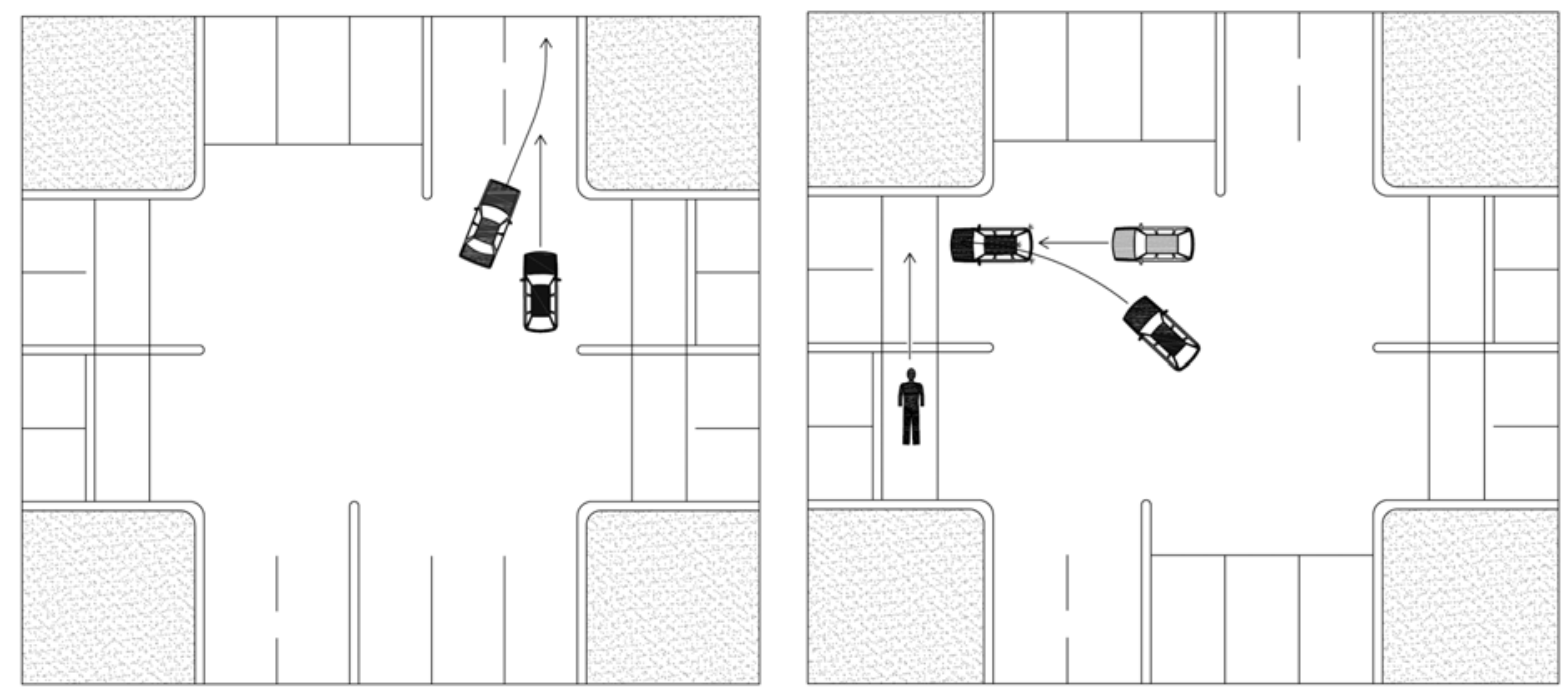

Figure 3: On the left is an example of one conflict between two vehicles - Lane change conflict;on the right is an example of secondary conflicts (from our catalogue 15 types of traffic conflicts).

However, some of these conflicts have very low rates of occurrence, which reduce their usefulness. The number of conflict types rises quickly when those that may occur between motorized and non-motorized road users are added to the list (pedestrians, cyclists, others). The list of conflict types that may be observed at a site depends upon its prevailing traffic rules and geometric characteristics; this list should be determined prior to initiating the study. It is not necessary to observe all traffic conflicts that may occur at a site in all conflict studies. If, for example, the objective is to compare the performance of two leftturn treatments at intersections, it might very well be sufficient to collect conflicts that are related to this manoeuvre. 


\section{d) How to conduct a traffic conflict study}

A number of elements need to be considered in the planning of a traffic conflict study:

- personnel training

- observation technique

- observation period

\section{Personnel training}

The validity and usefulness of a traffic conflict study are greatly influenced by the degree of consistency of the observers. Two basic requirements must be satisfied- the same observer must record conflicts consistently and different observers must record the same conflicts consistently.

\section{Observation technique}

The required number of observers (or the study duration when observations are made sequentially) depends on the number of conflict types to be observed, the average rate of occurrence for each conflict type, the volume of traffic, the number of intersection legs, and the need for a traffic count.

When all traffic conflict types need to be collected at a busy intersection, one observer will generally be able to watch only one intersection leg at a time. We can use mechanical or electronic counting devices (to facilitate data collection) or video camera, recording media, etc. (the recording of traffic conditions while conducting a conflict study is useful in later validating questionable conflicts or completing observations). Observations are generally made upstream from the location of the traffic conflicts of interest, in order to see vehicles' braking lights. Observers' exact location is influenced by the availability of space, the presence of obstructions to visibility, the types of conflicts to be observed, and the traffic speed. A distance of $30 \mathrm{~m}$ or more from the point of interest is usually adequate in urban areas (100m or more in rural areas). Observers should try to be inconspicuous to drivers to avoid modifying their behaviour. Appropriate locations include legal parking spaces, sitting behind a utility pole or a tree, etc. When allowed by the site configuration, observations should be made from an elevated point (e.g., the roof of a building) to avoid some visual obstructions (group of pedestrians, parked vehicles, etc.). When the observation period extends over several days, observers should try to maintain the same position throughout the study. When similar conflict studies need to be conducted at different sites, observers should try to use equivalent positions.

\section{Observation period}

In most cases, traffic conflict studies are conducted during daylight under dry weather conditions; observations should not be made under unusual conditions, such as road works, or special events that interfere with normal traffic patterns, unless justified by the need of the analysis.

- If accident analyses reveal a time-related pattern, observations should be planned when problems are the most likely to be observed (rush-hour periods, weekends, etc.).

- The observation period may vary from a few hours to several days, depending on the time needed to collect sufficient data. Typical observation periods range 
from between two to five days. Statistical methods have been developed to determine a study duration that will ensure a selected level of statistical reliability

- In order to help observers maintain a high level of concentration, a conflict study must be planned around sequences of observation periods and breaks. Parker and Zegeer (1988).

- If the vehicle intensity in peak hours is below $100 \mathrm{rv} / \mathrm{h}$ (reduction vehicle per hour), it is necessary to pursue metering longer than 1 hour.

\section{APPLICATION}

Before initiating the analysis, the observer must complete all the information on the traffic form heading to ensure that the location and observation conditions will be readily recognized in the future: municipality, intersection, approach, date, time, weather conditions and other comments.

Once observations have been completed, data must be reduced and summaries prepared. Results are presented either in summary tables or in traffic conflict diagrams (Figure 4). Summary tables allow comparisons of conflict rates between the site being analyzed and sites with similar characteristics, which is useful in detecting deviant patterns. The logic behind these analyses is similar to that of the accident pattern analysis. Traffic conflict diagrams are quite similar to the collision diagrams. They facilitate the identification of repetitive conflict patterns that are concentrated in some travel directions and intersection areas. For the likelihood of a confrontation of traffic conflicts between separate junctions the simple relative index of traffic conflicts $\mathbf{k}_{\mathbf{R}}$ was chosen.

$$
\mathbf{k}_{\mathbf{R}}=\left(\mathbf{P}_{\mathbf{k s}} / \mathbf{I}\right) * \mathbf{1 0 0} \text { [conflict situations } / 100 \text { reduction vehicles] }
$$

$\mathrm{P}_{\mathrm{ks}}$... conflict situations per hour (only conflict situations with a conflict severity 1-3), I... intensity rv/h (reduction vehicle per hour).

A pilot survey made in Děčín at the roundabout "Dělnická . Hankova - Ruská" with students of the Czech Technical University in Prague, Faculty of Transportation Science, Department of Transporting Systems (Figure 4). 


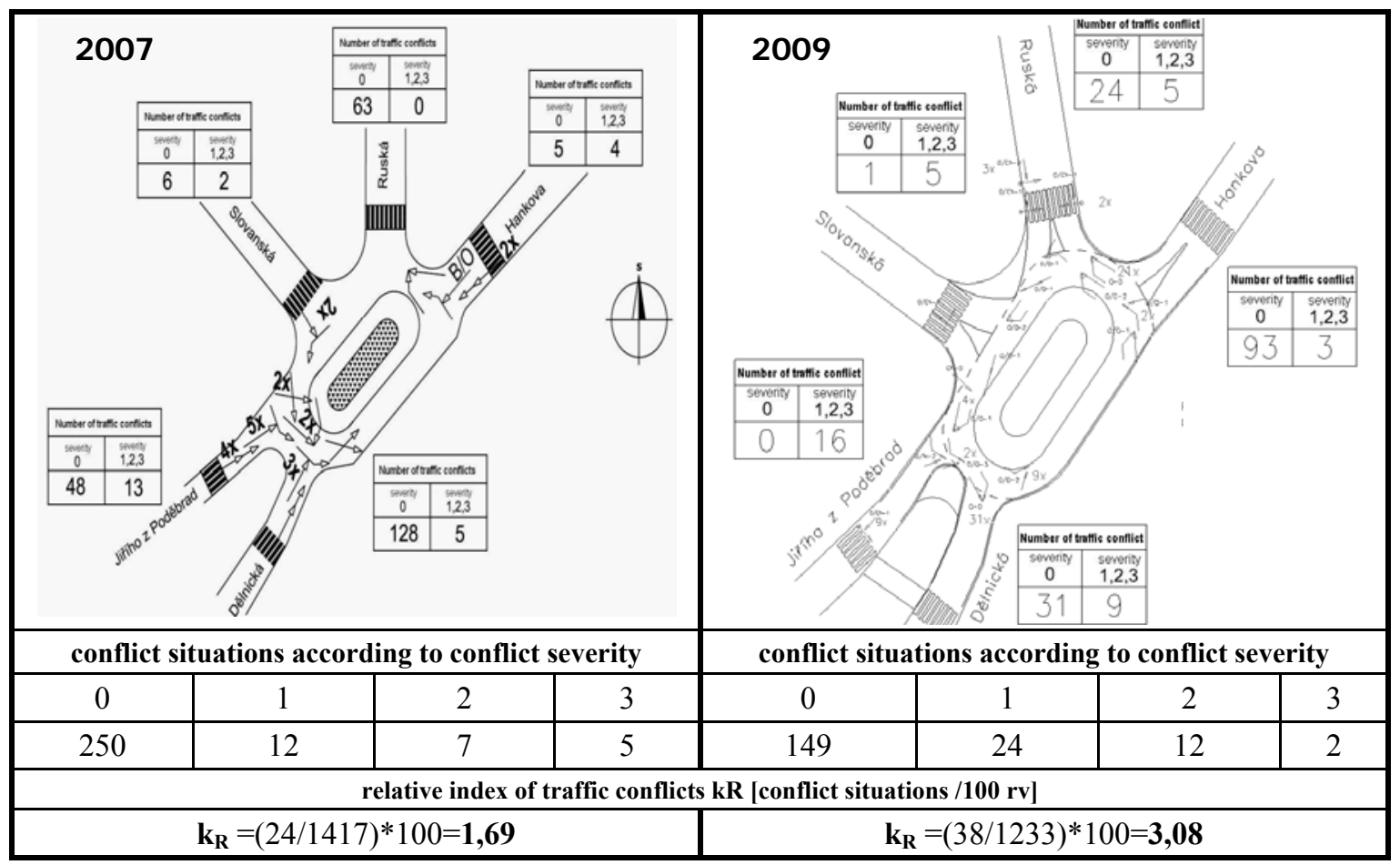

Figure 4: Example of one roundabout in Děčín. Junctions have a degrade relative index of traffic conflicts $(1.69-2007,3.08-2009)$. Relative index of traffic conflicts in year 2008 was 2.37 conflict situations $/ 100 \mathrm{rv}$.

\section{DISCUSSION AND CONCLUSION}

The article deals mainly with defining a way of monitoring traffic conflicts. This issue is only one part of a greater safety analysis, or the risk determination process of a particular location. Based on the described procedures it is also possible to apply the methodology to more complicated nodes such as level crossings, sections of towns, residential areas, or rural areas. After the survey and collection of the necessary amount of transport and engineering data, the determination of a relative number of "almost-accident rate" (the number of calculated vehicles for a selected time period and the number of traffic conflicts - so-called almost-accidents) may be calculated. At this stage, the overall risk result of the monitored node may not yet be stated. An important inter-stage is therefore, determination of an initial or safe state of a proved type of measure (e.g., ring crossings) and the deduction of generally valid hypotheses. The last step is the determination of a "risk parameter" of the problematic node. As a result, the crossing either passes or fails (the risk parameter is either good or bad). The part of the risk parameter will be dealt with by the Czech Technical University in Prague, Faculty of Transportation Science, Department of Transporting Systems, within its research task for the upcoming period.

\section{ACKNOWLEDGMENT}

For their cooperation in the traffic conflict survey many thanks are extended to the employees and students of the Czech Technical University in Prague, Faculty of Transportation Science, Department of Transporting Systems. My contribution was supported by the project of the Ministry of Education, Youth and Sports No. MSM 6840770043. 


\section{REFERENCES}

Road Safety Manual, Recommendations from the World Road Association PIARC (2003).

Parker, M. Jr. - Zegeer, C.: Traffic Conflict Techniques For Safety And Operations, Observal Manual, (2003).

Kocourek, J.: The Road Safety Traffic in Dependencies on Traffic Terms and Constructional Form on Communication. 2008, Academic dissertation on the Czech Technical University in Prague Faculty of Transportation Science Department of Transporting Systems. 